\title{
Uma Nova Função de Aproximação Least Square com Zeros Imaginários Obtidos por Otimização
}

\author{
Calisto Schwedersky e Sidnei Noceti Filho
}

Resumo: Neste trabalho, é estudada uma classe especial de funções de aproximação que utiliza o método least-squares, o qual confere mínima perda de potência na banda de passagem. Esse método permite a escolha de algumas peculiaridades, tais como monotonicidade ou ripples variáveis; ênfase para algumas freqüências na banda de passagem; escolha da banda a ser minimizada e inclusão de zeros imaginários. Também é apresentada uma nova função de aproximação (SN), que utiliza uma função característica least-squares em que se aplica novamente o mesmo método para otimizar a banda de rejeição. Essa otimização produz zeros imaginários, os quais tornam a função mais próxima de um brick-wall filter.

Palavras-chave-Filtros passa-baixa, funções de aproximação, pólos e zeros.

Abstract: In this paper, a special class of approximation functions is studied using the least-squares method, which yields a minimal of power loss in passband. This method allows the choice of some proprieties, such as either monotonic or nonmonotonic, weighting of some passband frequencies, band choice to be minimized, and inclusion of imaginary zeros. In addition, a new approximation function (SN) is presented, which uses a least-squares characteristic function, applying again the same method to optimize the bandstop. This optimization provides imaginary zeros, which makes the obtained function nearest of a brick-wall filter.

Keywords-Low-pass filters, approximation functions, poles and zeros.

\section{INTRODUÇ̃̃O}

Uma importante classe de Funções de Aproximação de Filtros são as chamadas Least-Square Approximations (LS). Elas proporcionam o melhor compromisso entre as perdas na banda de passagem e as perdas na banda de rejeição, pois são obtidas minimizando (com ou sem uma função de ponderação) a razão entre a potência refletida e a potência transmitida, em temos da norma least-mean-square.

As Funções LS podem ser classificadas com relação à localização dos zeros e ao comportamento da resposta em freqüência na banda de passagem e na banda de rejeição:

A) Funções polinomiais (zeros no infinito), com ripples na banda de passagem

B) Funções polinomiais (zeros no infinito), monotônicas na banda de passagem

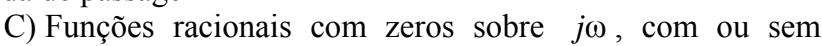
ripples na banda de passagem

Uma das primeiras funções LS foi proposta por Pottle e Wong [1] (PW), onde a integral de minimização é feita diretamente na função de transferência do filtro $T(s)=1 / H(s)$, onde $H(s)$ é a

Calisto Schwedersky e Sidnei Noceti Filho, LINSE - Laboratório de Circuitos e Processamento de Sinais, Depto. de Eng. Elétrica, Universidade Federal de Santa Catarina, Florianópolis, SC, E-mails: \{calisto, sidnei\}@linse.ufsc.br.

Este trabalho foi parcialmente financiado pelo $\mathrm{CNPq}$ chamada função atenuação. No entanto, foram obtidos ripples relativamente altos na banda de passagem e as características na banda de rejeição se mostraram relativamente ruins (menos seletivas).

Humpherys [2] (HU) propôs realizar a minimização na função característica $Q_{n}(\omega)$. Ela se relaciona com $H(\omega)$ segundo (1):

$$
|H(\omega)|^{2}=1+\varepsilon^{2} Q_{n}^{2}(\omega) \text { ou } H(s) H(-s)=1+\left.\varepsilon^{2} Q_{n}^{2}(\omega)\right|_{\omega^{2}=-s^{2}}
$$

A função atenuação em dB é dada por:

$$
A(\omega)=|H(\omega)|_{\mathrm{dB}}=10 \log _{10}\left(1+\varepsilon^{2} Q_{n}^{2}(\omega)\right) .
$$

No limite da banda passante normalizada, tem-se $A(1)=A_{\max }=10 \log _{10}\left(1+\varepsilon^{2} Q_{n}^{2}(1)\right)$. Quando a função é normalizada $\left(Q_{n}(1)=1\right)$, então $\varepsilon=\left(10^{0,1 \text { Amax }}-1\right)^{1 / 2}$ [3].

Com o uso de continuação analítica (teoria de variáveis complexas), ao se substituir $\omega$ por $s / j$, obtém-se a equação de Feldtkeller [3]. Após encontrar os zeros de $H(s) H(-s)$ (pólos da função de transferência), escolhe-se aqueles cuja parte real é negativa, já que o filtro deve ser estável.

O método LS tem como base a utilização de (2):

$$
E=\int_{\omega_{1}}^{\omega_{2}} w(\omega) Q_{n}^{2}(\omega) \partial \omega .
$$

A minimização desta integral possibilita a obtenção de uma menor perda de potência na faixa de freqüências $\left(\omega_{1}, \omega_{2}\right)$ desejada. A função $w(\omega)$ é utilizada para ponderar $Q_{n}(\omega)$ em certas freqüências. Ela pode ser simplesmente igual a 1 (caso mais comum), $\quad w(\omega)=\omega^{2}, \quad w(\omega)=\left(1-\omega^{2}\right)^{2}, \quad w(\omega)=c \omega^{c-1}$ (Rakovich [4]), $w(\omega)=\left(1-\omega^{2}\right)^{\lambda-0,5}$ (Rakovich e Popovich [5] (RO)), ou uma forma mais geral $w(\omega)=\omega^{a}\left(1-\omega^{2}\right)^{b}$ (Phuc e Attikiouzel [6]). A Fig. 1 mostra a função estudada em [6] para alguns valores de $a$ e $b$.

$\mathrm{O}$ intervalo de integração mais comum compreende toda a banda de passagem, sendo $\omega_{1}=0$ e $\omega_{2}=1$ para a banda normalizada. O uso de limites diferentes destes é discutido adiante.

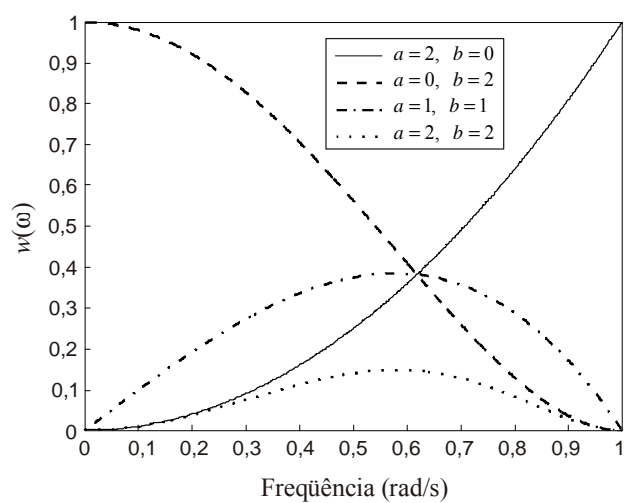

Fig. 1. Função $w(\omega)=\omega^{a}\left(1-\omega^{2}\right)^{b}$ para vários valores de $a$ e $b$. 
Neste trabalho, é proposta uma nova função de aproximação (SN) que apresenta características vantajosas em alguns aspectos. A nova função SN consiste em adicionar zeros otimizados sobre $j \omega$ na função polinomial $\mathrm{HU}$, tornando-a mais seletiva na banda de transição. A função HU apresenta a magnitude que mais se aproxima do brick-wall (filtro passa-baixa ideal) na banda de passagem. Com a adição de zeros sobre $j \omega$, são melhoradas as características de atenuação e, também, ocorre uma pequena melhora na banda de passagem em relação à função HU.

Dois métodos de colocação de zeros em funções LS são encontrados na literatura. Nos dois, os zeros têm seus valores pré-definidos. No primeiro método, os zeros escolhidos são considerados no cálculo de $Q_{n}(\omega)$ utilizando (2). Isso é feito, por exemplo, em Rakovich e Popovich [5] e Phuc e Attikiouzel [7] (PK). No segundo método, os zeros escolhidos são introduzidos na função já calculada $Q_{n}(\omega)$ como, por exemplo, em Rakovich e Litovski [8] (LK).

No método proposto, é realizada a integral de minimização na banda de rejeição, sendo encontrados os zeros que proporcionam uma maior atenuação na banda de rejeição (faixa de integração). Exemplos são mostrados onde a nova função é comparada com outras existentes na literatura.

\section{REVISÃo dOS VÁRIOS MÉTOdos LS}

\section{A. Funções Polinomiais com Ripples na Banda de Passagem}

Uma função de transferência $T(s)$ polinomial (todos os zeros de transmissão no infinito) é gerada por uma função característica $Q_{n}(\omega)$ também polinomial. Diferentes tipos de $Q_{n}(\omega)$ geram ripples em $|T(\omega)|$. Uma das primeiras destas funções, mostrada em (3), foi estudada por Humpherys [2] (HU), por Picciarelli e Torre [9] (PT) e Thajchayapong [10] (TH):

$$
Q_{n}(\omega)=\left\{\begin{array}{l}
a_{n} \omega^{n}+a_{n-2} \omega^{n-2}+\ldots+a_{0}, \text { n par } \\
a_{n} \omega^{n}+a_{n-2} \omega^{n-2}+\ldots+a_{1} \omega, n \text { ímpar }
\end{array} .\right.
$$

Outros tipos de $Q_{n}(\omega)$ foram propostos por Popovich [11] (PO), Jovanović e Rabrenović [12] (JR) e Phuc e Attikiouzel [7, 13, 14, 15] (PK).

A função PK utiliza $Q_{n}(\omega)$ da seguinte forma:

$$
Q_{n}(\omega)=\omega^{l} \sum_{i=0}^{m} a_{2 i} \omega^{2 i}, \quad l+m=n .
$$

Para $l=0$ e $l=1$, obtém-se a função HU para $n$ par e ímpar, respectivamente. $\mathrm{O}$ parâmetro $m$ determina o número de oscilações na banda de passagem. O caso específico de $m=2$ (duas oscilações) foi estudado por Thajchayapong e Lomtong [16] (LT). O caso específico de $m=1$ foi estudado em JR [12].

A Fig. 2 mostra funções PK de ordem 5 para diferentes valores de $m$. Quando $m=0$, obtém-se um filtro Butterworth (BT) maximamente plano na banda de passagem, mas com uma menor seletividade. Quando $m=2$, obtêm-se a função HU que possui a maior seletividade e também um ripple menor do que no caso $m=1$. Para o caso da função PO, tem-se:

$$
Q_{n}(\omega)=\omega^{l}\left(\frac{\omega^{2}-\omega_{0}^{2}}{1-\omega_{0}^{2}}\right)^{2 m}, \quad l+m=n .
$$

A função PO apresenta curvas de magnitude com apenas uma oscilação, independentemente da ordem n. A Fig. 3 mostra funções PO de ordem 7 para diferentes valores de $m$. Quando $m=2$, obtém-se a maior atenuação na banda de rejeição juntamente com um ripple um pouco maior do que no caso $m=3$. Quando $m=0$, obtém-se um filtro BT (com menor seletividade). Quando $m=1$, o ripple é alto e a sua característica na banda de rejeição não é tão boa quanto nos casos $m=2 \mathrm{e}$ $m=3$.

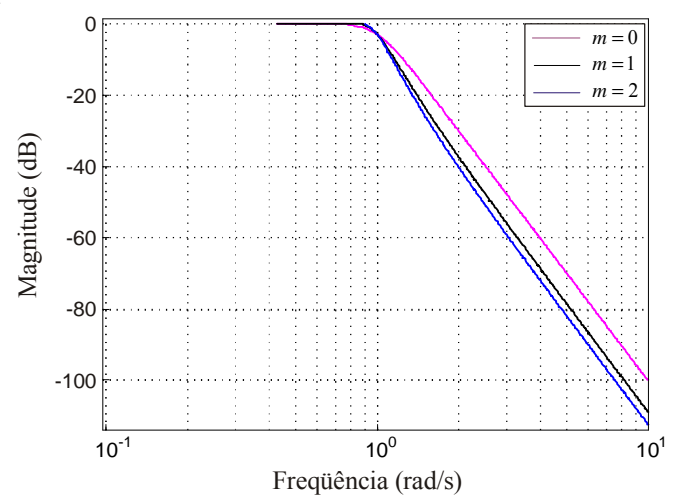

(a)

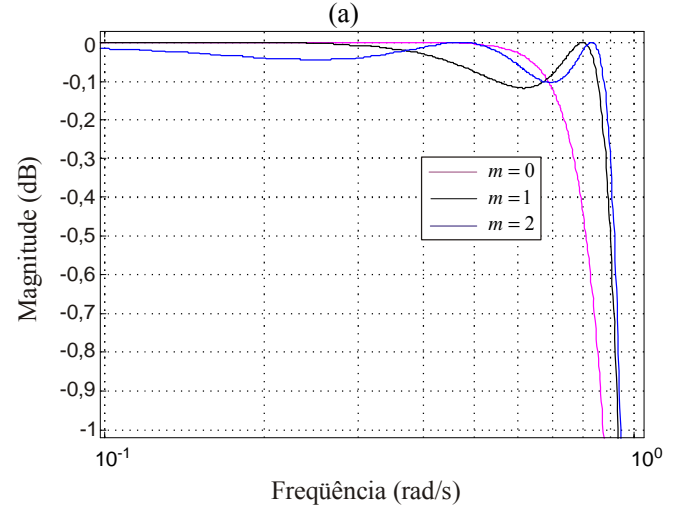

(b)

Fig. 2. (a) Magnitude das Funções PK; (b) zoom na banda de passagem.

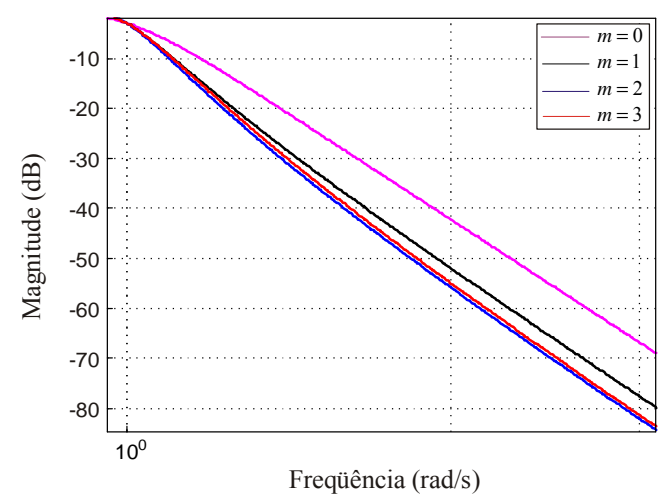

(a)

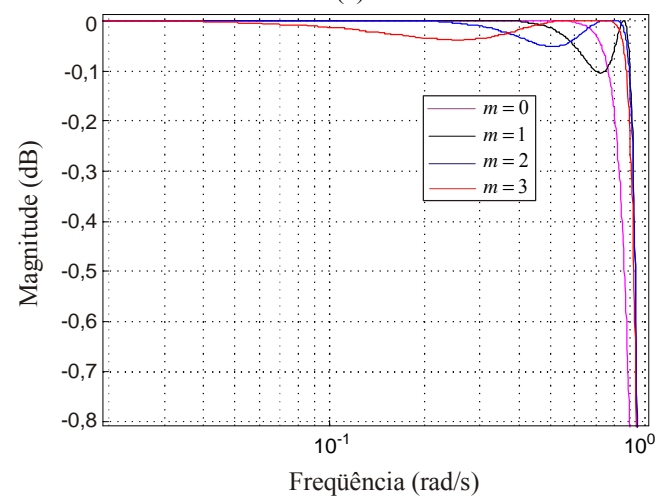

(b)

Fig. 3. (a) Magnitude das Funções PO; (b) zoom na banda de passagem. 
Observa-se que funções com ripple apresentam uma maior seletividade quando comparadas com funções monotônicas. As funções PO e PK, quando $m=0$, geram a função BT. Quando $m=1$, geram a função JR que possui apenas uma oscilação e um coeficiente a ser calculado, com fácil solução analítica. A função PK permite obter quase todos os tipos de $Q_{n}(\omega)$ com ripples (JR, HU, LT), com exceção da função PO.

Para determinar a função característica do filtro PO, utiliza-se a propriedade $\partial E / \partial \omega_{0}=0$ na integral (2). Essa propriedade é usada para se obter uma freqüência $\omega_{0}$ que minimize a integral. Na equação obtida, $\omega_{0}$ é facilmente encontrada.

No caso da função $\mathrm{PK}, Q_{n}(\omega)$ é determinado utilizando a propriedade $\left(\partial E / \partial a_{i}\right)=0$ na integral (2). Esta propriedade gera um conjunto de equações lineares. Para evitar que a solução do sistema de equações seja $a_{i}=0$, são necessárias condições de realizabilidade. A mais utilizada é $Q_{n}(1)=1$ (fazendo com que $\sum a_{i}=1$ e assim $|H(j 1)|_{\mathrm{dB}}=-3 \mathrm{~dB}$, ou seja, $\left.\varepsilon=1\right)$.

Além da condição $Q_{n}(1)=1$, Malvar e Calôba [17] (CM) utilizaram também as condições $\partial Q_{n}(\omega) /\left.\partial \omega\right|_{\omega=1}=n^{2}$ e $a_{n}=2^{n-1}$, obtendo filtros com comportamento na banda de rejeição semelhante ao dos filtros Chebyshev (CB). A Fig. 4 mostra uma comparação entre esses três tipos de condições. As curvas em azul e vermelho foram normalizadas para poderem ser comparadas com a curva em preto (que é normalizada). Percebe-se que, com o aumento do ripple, obtém-se maior seletividade na banda de rejeição, porém a curva em preto é a que proporciona a maior transferência de potência na banda de passagem, isto é, apresenta menores perdas.
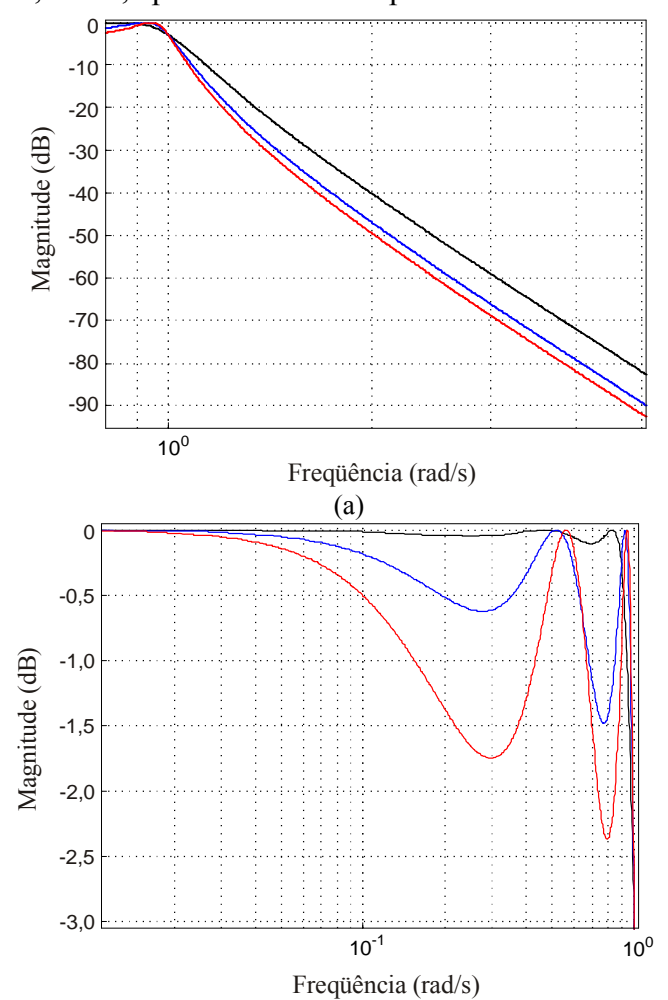

(b)

Fig. 4. (a) Magnitude das funções PK utilizando diferentes restrições: $Q_{n}(1)=1 \quad$ (preto), $\left.\quad\left(\partial Q_{n}(\omega) / \partial \omega\right)\right|_{\omega=1}=n^{2} \quad($ azul $) \quad$ e $\quad a_{n}=2^{n-1}$ (vermelho); (b) zoom na banda de passagem.

\section{B. Funções Polinomiais Monotônicas na Banda de Passagem}

Rakovich e Litovski [18] (LR) utilizaram o método LS para obter funções monotônicas, utilizando $Q_{n}(\omega)$ na forma:

$$
\begin{gathered}
Q_{n}^{2}(\omega)=\int_{0}^{\omega} x\left[\sum_{i=0}^{p} C_{i} U_{i}(x)\right]^{2} \partial x, \text { onde } p=\left\{\begin{array}{l}
n / 2-1, n \text { par } \\
(n-1) / 2, n \text { ímpar }
\end{array}\right. \\
\text { e } \quad U_{i}(x)=2 \sqrt{i+1} \sum_{k=0}^{i} \frac{(-1)^{k}(2 i+1-k) ! x^{2(i-k)+1}}{k !(i-k) !(i-1+k) !}, n \text { par } \\
U_{i}(x)=\sqrt{4 i+2} \sum_{k=0}^{i} \frac{(-1)^{i-k}(i+k) ! x^{2 k}}{(k !)^{2}(i-k) !}, n \text { ímpar. }
\end{gathered}
$$

Os polinômios $U_{i}(x)$, que são derivados dos polinômios de Jacobi deslocados $G(p, q, y)$ [19] através de uma mudança de variável, são ortogonais em relação à função peso $w(x)=x$ no intervalo $0 \leq x \leq 1$. Desde que todos os zeros de $U_{i}(x)$ são simples e estão no intervalo de ortogonalidade (que também é a banda de passagem do filtro), as raízes da primeira derivada de $Q_{n}^{2}(\omega)$ têm multiplicidade par e não mudam de sinal, garantindo assim a monotonicidade da função.

A Fig. 5 mostra as magnitudes das funções LR de ordens 4, 5 e 6 que apresentam pontos onde a derivada é zero.

Para a obtenção de $Q_{n}(\omega)$ das funções LR, a integral a ser minimizada é a mesma de (2) com os mesmos graus de liberdade para $\omega_{1}, \omega_{2}$ e $w(\omega)$.

As condições $\partial E / \partial C_{i}=0$ e $Q_{n}(1)=1$ (que implica em $\left.\sum C_{i}^{2}=1\right)$ são utilizadas para calcular os coeficientes $C_{i}$. Após algumas manipulações algébricas, obtém-se o seguinte conjunto de equações:

$$
\begin{gathered}
\sum C_{i}^{2}=1 \\
C_{j} J_{j+1}-C_{j+1} J_{j}=0, \quad j=0,1, \ldots,(p-1)
\end{gathered}
$$

onde:

$$
J_{j}=\frac{1}{2 C_{j}} \frac{\partial E}{\partial C_{j}}=\int_{\omega_{1}}^{\omega_{2}} w(\omega) \int_{0}^{\omega} x U_{j}(x)\left[\sum_{i=0}^{p} C_{i} U_{i}(x)\right] \partial x \partial \omega .
$$

Neste caso, obtém-se um conjunto de equações não lineares, tornando-se necessária a utilização de métodos numéricos para solucionar o sistema.

Na Fig. 6, é mostrada uma comparação, para ordem 7, da função LR com a função BT. Nota-se que a função LR é mais seletiva na banda de rejeição.

C. Funções Racionais com Zeros sobre jw, com ou sem Ripples na Banda de Passagem

Para o caso das funções não-monotônicas, Kurtz, Orchard e Temes [20] foram uns dos primeiros a estudar a introdução de zeros nos filtros LS, com $Q_{n}(\omega)$ racional. O método é praticamente o mesmo para todos os tipos de função, seja PK, PO ou LR. Assim, no caso da função PK com pares de zeros no eixo imaginário, $Q_{n}(\omega)$ é:

$$
Q_{n}(\omega)=\frac{N_{n}(\omega)}{D_{K}(\omega)}=\frac{\omega^{l} \sum_{i=0}^{m} a_{2 i} \omega^{2 i}}{\left(\omega^{2}-\omega_{1}^{2}\right) \ldots\left(\omega^{2}-\omega_{K}^{2}\right)}
$$

onde $l+m=n$ e $K \leq \begin{cases}n / 2, & n \text { par } \\ (n-1) / 2, & n \text { ímpar }\end{cases}$ 
e a variável $K$ representa o número de pares de zeros do filtro. A função $|T(\omega)|^{2}$ é:

$$
|T(\omega)|^{2}=\frac{\left(\omega^{2}-\omega_{1}^{2}\right)^{2} \ldots\left(\omega^{2}-\omega_{K}^{2}\right)^{2}}{\left[\left(\omega^{2}-\omega_{1}^{2}\right)^{2} \ldots\left(\omega^{2}-\omega_{K}^{2}\right)^{2}\right]+\alpha \varepsilon^{2} N_{n}^{2}(\omega)}
$$

onde $\alpha=\left(\omega_{1}^{2}-1\right)^{2} \ldots\left(\omega_{K}^{2}-1\right)^{2}$. A constante $\alpha$ é utilizada para garantir que a função seja normalizada, isto é, $|T(1)|_{\mathrm{dB}}=-3 \mathrm{~dB}$ (para $\varepsilon^{2}=1$ ).

Para o caso das funções PO, o polinômio $Q_{n}(\omega)$ é:

$$
Q_{n}(\omega)=\frac{N_{n}(\omega)}{D_{K}(\omega)}=\frac{\omega^{l}\left(\frac{\omega^{2}-\omega_{0}^{2}}{1-\omega_{0}^{2}}\right)^{2 m}}{\left(\omega^{2}-\omega_{1}^{2}\right) \ldots\left(\omega^{2}-\omega_{K}^{2}\right)} .
$$

Assim, $|T(\omega)|^{2}$ passa a ser:

$$
|T(\omega)|^{2}=\frac{D_{K}^{2}(\omega)}{D_{K}^{2}(\omega)+\varepsilon^{2} N_{n}^{2}(\omega)} .
$$

Para as funções PK e PO, é realizada a integral de minimização (2) utilizando os polinômios $Q_{n}(\omega)$ apresentados em (12) e (14), respectivamente.

Para o caso das funções monotônicas, foi mostrado por Rakovich e Litovski [8] (LK) que a utilização do polinômio dos zeros, $D_{K}(\omega)$, na integral de minimização (2), praticamente não altera os coeficientes $C_{i}$ da função LR [18], além de aumentar muito a complexidade do cálculo. Assim, $|T(\omega)|^{2}$ é o mesmo da equação (13) e a integral (2) é feita utilizando $N_{n}(\omega)$ ao invés de $Q_{n}(\omega)$, descartando o polinômio $D_{K}(\omega)$ do cálculo.

\section{INFLUÊNCIA DOS LIMITES DE INTEGRAÇ̃̃o}

Rakovich [21] e também Djurich e Petkovich [22] mostraram que, conforme os valores de $\omega_{1}$ e $\omega_{2}$, pode-se obter os filtros monotônicos BT, Papoulis-Fukada (PF), LR e Halpern (HA).

Quando $\omega_{1}=0 \mathrm{e} \quad \omega_{2} \rightarrow 0$, é obtido um BT. Isso ocorre porque, nessas condições, valoriza-se a máxima planitude na origem. Quando $\omega_{1} \rightarrow 0 \mathrm{e} \quad \omega_{2}=1$, é obtido um filtro PF que possui, na freqüência limite da banda passante, a maior derivada na característica de magnitude. Quando $\omega_{1}=1$ e $\omega_{2} \rightarrow \infty$, obtémse um filtro HA que possui o corte mais abrupto (a partir de uma freqüência próxima ao limite da banda de passagem).

Existe outra maneira de obter os filtros HA, PF e LR através de uma mudança no valor de uma constante $p$, método que foi mostrado por Rakovich e Popovich [23].

\section{Comparação Entre Funções LS}

A Fig. 7 mostra uma comparação dos filtros $\mathrm{PO}(m=3)$, PK $(m=3)$ e LR de ordem 6. Percebe-se que o fato do filtro PO possuir apenas uma oscilação não traz vantagens, visto que o ripple é grande e sua atenuação é menor. A função LR tem a vantagem de ser monotônica, porém a função que possui a maior seletividade é a função PK.

\section{MÉtodo Proposto}

Para um dado $Q(\omega)$, o método proposto consiste em acrescentar na função $|T(\omega)|^{2}$ um polinômio $D_{K}^{2}(\omega)$ (ver (16)), cujos coeficientes serão calculados através do método LS. Isso resulta em um $T(s)$ com $K$ pares de zeros sobre $j \omega$, aumentando a seletividade na banda de transição.
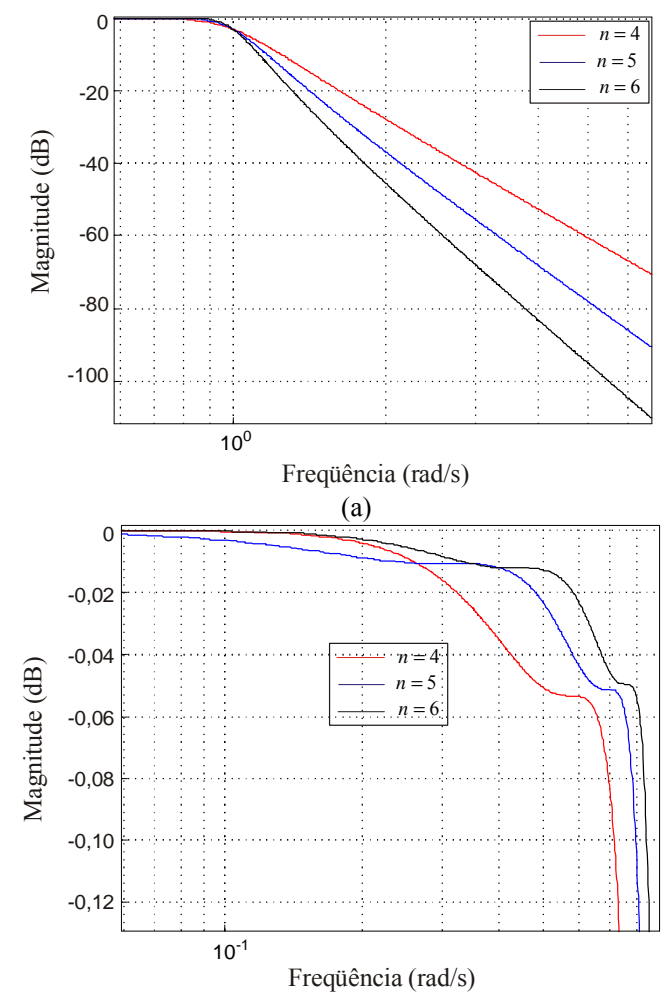

(b)

Fig. 5. (a) Magnitude das funções LR; (b) zoom na banda de passagem.

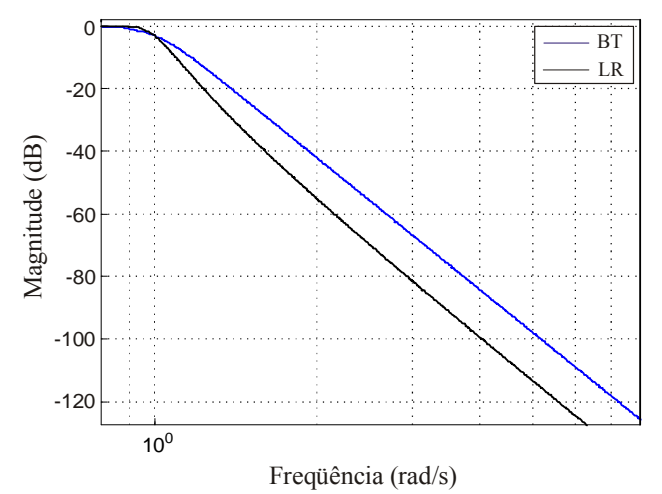

(a)

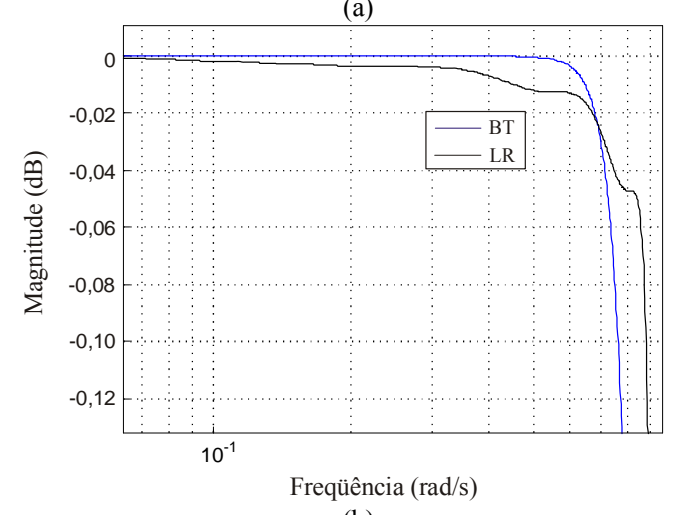

(b)

Fig. 6. (a) Magnitude das Funções BT e LR de ordem 7; (b) zoom na banda de passagem.

A função tem a forma:

$$
|T(\omega)|^{2}=\frac{1}{1+\varepsilon^{2} \frac{Q_{n}^{2}(\omega)}{D_{K}^{2}(\omega)}}, \quad K \leq \begin{cases}n / 2 & n \text { par } \\ (n-1) / 2 & n \text { ímpar }\end{cases}
$$


O polinômio $D_{K}(\omega)$ é dado por:

$$
D_{K}(\omega)=\sum_{i=0}^{i=K} \omega^{2 i} b_{2 i}
$$

Os coeficientes $b_{2 i}$ são determinados visando a minimização da integral de $D_{K}^{2}(\omega) / Q_{n}^{2}(\omega)$, que resultará em uma maior derivada de $|T(\omega)|$ após a freqüência limite da banda passante. A condição dada por (18) visa a manutenção de uma desejada atenuação $A_{\max }$ no limite da banda $\left(A_{\max }=f(\varepsilon)\right)$.

$$
D_{K}(1) / Q_{n}(1)=1
$$

$$
\text { Assim, } \quad b_{2 K}=1-\sum_{i=0}^{i=K-1} b_{2 i} \text {. }
$$

Este valor de $b_{2 K}$ é então substituído em $D_{K}(\omega)$. Após, é efetuada a integração dada por (20), onde $\omega_{1}$ e $\omega_{2}$ são os limites extremos da banda de rejeição

$$
E=\int_{\omega_{1}}^{\omega_{2}} \frac{D_{K}^{2}(\omega)}{Q_{n}^{2}(\omega)} \partial \omega
$$

O limite $\omega_{2}$ não precisa ser infinito. Resultados obtidos por simulação mostram que, a partir de $\omega_{2}>10 \omega_{1}$, os coeficientes $b_{2 i}$ praticamente não se alteram. Para o caso do limite $\omega_{1}$, quanto menor seu valor, maior é a seletividade na banda de transição, mas pior será a seletividade na banda de rejeição (ver Fig. 8).

Após, faz-se as derivadas da integral com relação aos coeficientes $b_{2 i}$ (com exceção de $b_{2 K}$, que é calculado posteriormente). Igualando as derivadas a zero, encontra-se o ponto de mínimo. As derivadas a serem utilizadas são dadas por (21).

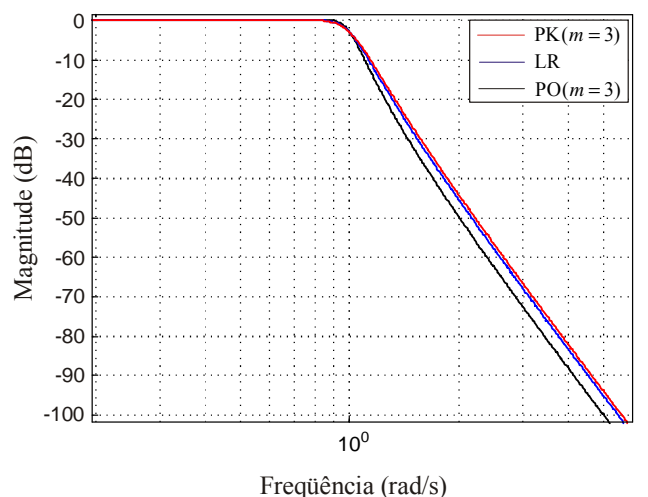

(a)

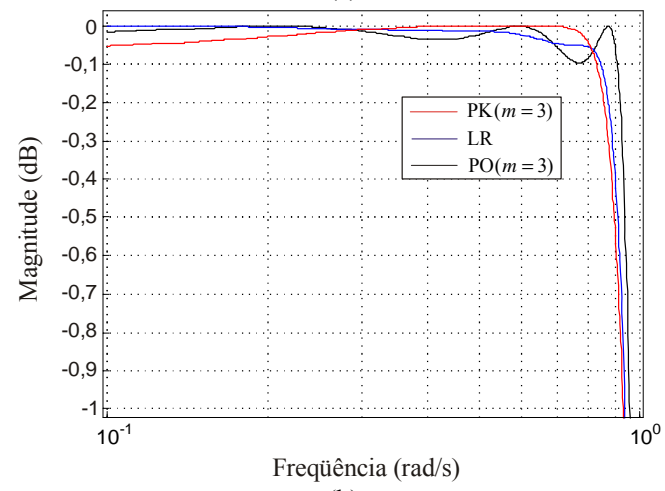

(b)

Fig. 7. (a) Magnitude das funções PK, PO e LR; (b) zoom na banda de passagem.
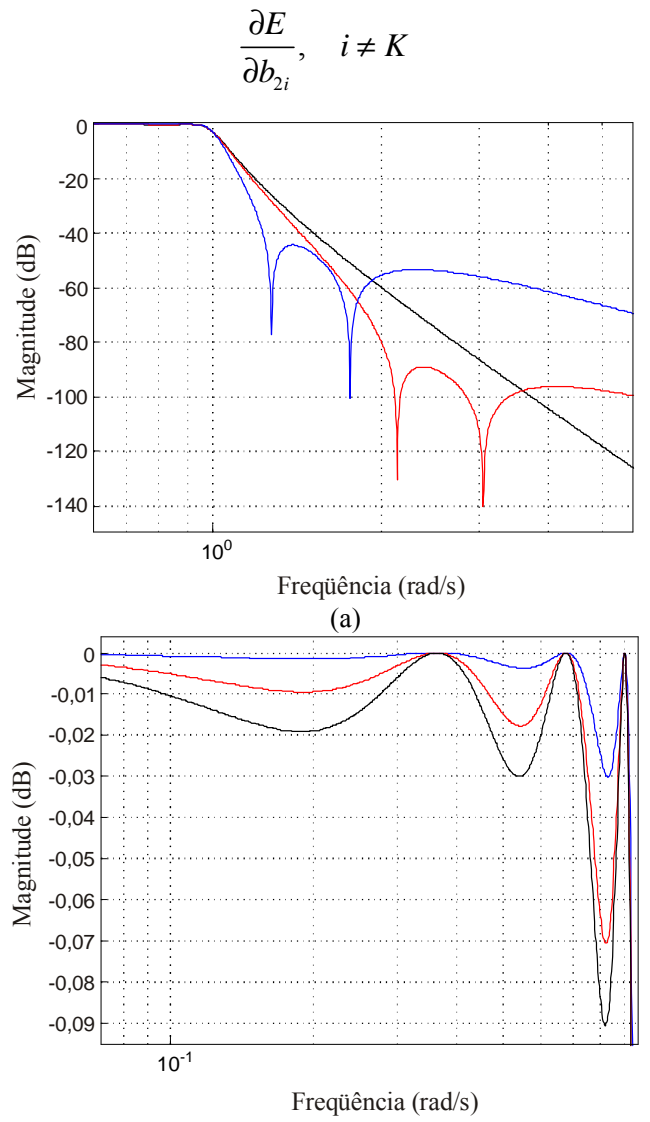

(b)

Fig. 8. (a) Magnitude das funções HU (preto) e SN $(K=2)$, para $\omega_{1}=1,2$ (azul) e para $\omega_{1}=2$ (vermelho); (b) zoom na banda de passagem.

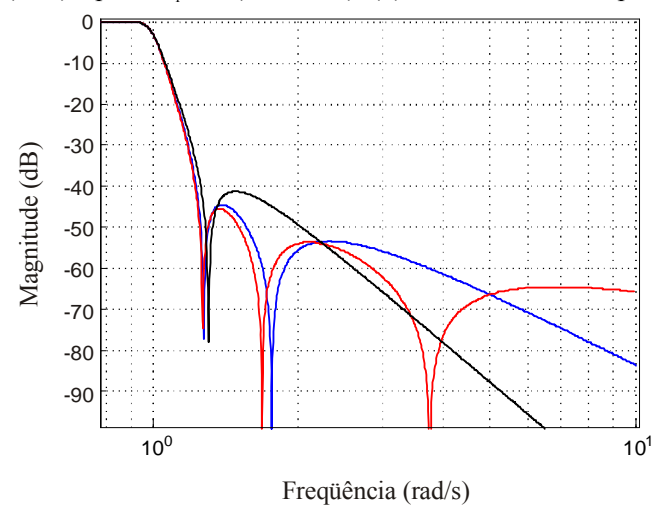

(a)

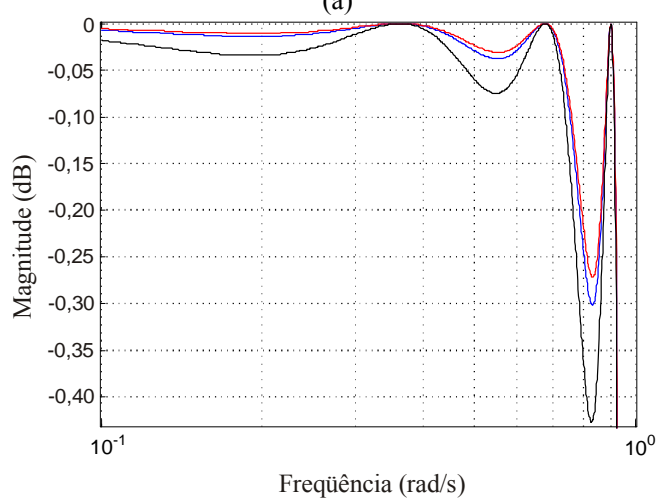

(b)

Fig. 9. (a) Magnitude das funções SN para $K=1$ (preto), $K=2$ (azul) e $K=3$ (vermelho), usando $\omega_{1}=1,2$ e $\omega_{2}=10$; (b) zoom na banda de passagem. 
Utilizando (21), obtém-se um conjunto de equações lineares que depois de resolvido fornece os coeficientes $b_{2 i}$. O coeficiente $b_{2 K}$ é então calculado através de (19).

A Fig. 8 compara os filtros $\mathrm{HU}$ e $\mathrm{SN}$ de ordem 7 para diferentes valores de $\omega_{1}$. Percebe-se que os filtros SN apresentam um ripple um pouco menor e um corte mais abrupto.

A Fig. 9 mostra os novos filtros $\mathrm{SN}$ de ordem 7 para $K=1$, $K=2$ e $K=3$. Com o aumento do número de pares de zeros, o ripple da função diminui, o corte se torna mais abrupto e a atenuação na banda de rejeição aumenta.

A Fig. 10 mostra uma comparação entre os filtros SN, Cauer e Chebyshev Inverso para ordem 7 e $A_{\min }=91,5 d B$. O filtro SN tem um comportamento melhor do que o Cauer com relação ao ripple na banda de passagem, e melhor do que o Chebyshev Inverso com relação à seletividade.

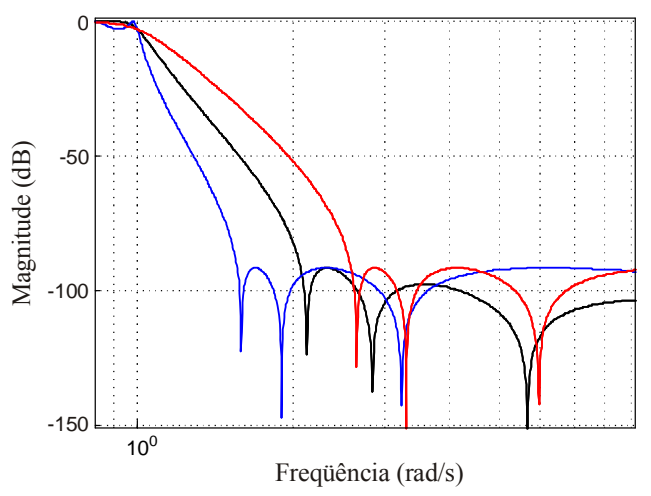

(a)

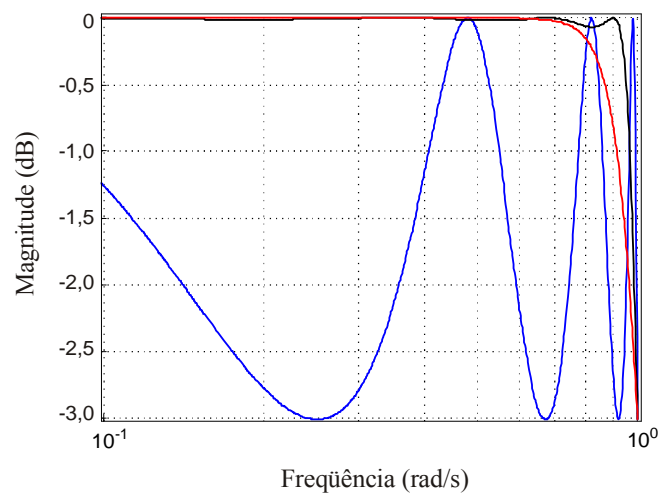

(b)

Fig. 10. (a) Magnitude das funções $\mathrm{SN}\left(K=3, \omega_{1}=2\right.$ e $\left.\omega_{2}=10\right)$ (preto), Cauer (azul) e Chebyshev Inverso (vermelho); (b) zoom na banda de passagem.

\section{CONCLUSÕES}

Neste trabalho, foram apresentadas diversas funções obtidas através do método least-squares. Foi mostrado também todo o procedimento para obtenção dessas funções. Essas funções têm a vantagem de possuírem perdas minimizadas na banda de passagem. Observa-se que as funções LS com ripples são as funções que possuem a maior seletividade dentre todas as funções LS. Também foi apresentada uma nova função de aproximação, a SN, que, através da utilização do método leastsquares aplicado na otimização da banda de rejeição, proporciona uma seletividade maior quando comparada com as funções da qual foi originada, além de menores ripples. Quando comparada com a função Chebyshev Inverso de mesma ordem e atenuação na banda de rejeição, a nova função apresenta o melhor desempenho na banda de passagem (menores perdas) e na banda de transição (maior seletividade).

\section{REFERÊNCIAS}

[1] . C. Pottle e J. Wong, "Optimum least-squares approximations to the ideal low-pass filter", IEEE Transactions on Circuit Theory vol. 17, no. 2, pp. 282-284, 1970 .

[2] D. S. Humpherys, "The analysis, design and synthesis of electrical filters", Prent-Hall, chap. 6, pp. 380-385, 1970.

[3] S. Noceti Filho, "Filtros Seletores de Sinais", EDUFSC, 3- Ed., 2009.

[4] B. D. Rakovich, "Using least-mean-square-approximation technique in filter design", International Journal of Circuit Theory \& Applications vol. 5, no. 2, pp.171-179, 1977.

[5] B. Rakovich e M. Popovich, "Characteristic function of least-meansquare Passband filters with finite attenuation poles", IEEE CAS vol. 27, no. 12, pp.1225-1233, 1980.

[6] D. T. Phuc e J. Attikiouzel, "All-pole filter designs using Lagrangian multipliers", Int. Journal of Elec. vol. 47, no. 5, pp. 491-501, 1979

[7] D. T. Phuc e J. Attikiouzel, "General analysis of Nonmonotonic Lowpass filters having multiple pairs of imaginary-axis zeros with weighted least-mean-square error charactc", El L vol. 14, no. 18, pp.600-602, 1978 .

[8] B. Rakovich e V. Litovski, "Monotonic Passband low-pass filters with Chebyshev Stopband attenuation", IEEE Transactions on ASSP, vol. 22, no. 1, pp.39-44, 1974.

[9] V. Picciarelli e M. Torre, "Synthesis of filters with minimum integrated power-loss ratio in the Passband", Electronics Letters vol. 13, no. 20, pp.612-614, 1977.

[10] P. Thajchayapong, "Least-mean-square (minimum integrated power loss ratio) Lowpass filters with a pair of imaginary- axis zeros", Elect. Letters vol. 14, no. 9 pp.268-270, 1978.

[11] M.V. Popovich, "Selective filters using least-mean-square approximation technique", Electronics Letters vol. 14, no. 18, pp.596597,1978

[12] V. Jovanović e D. Rabrenović, "Lowpass-filter flat magnitude characteristic with sharp cutoff", Electronic Letters vol.11, no. 8, pp. $174,1975$.

[13] D. T. Phuc e J. Attikiouzel, "A sharp cut-off filter with a controlled Passband”, Int. Journal of Elect. vol. 47, no. 3, pp. $285-295,1979$.

[14] D. T. Phuc e J. Attikiouzel, "Lowpass filters having multiple pairs of imaginary-axis zeros at two distinct points in the Stopband with weighted L.M.S. error characteristic", Elect Letters vol. 14, no. 22, pp.706-708, 1978.

[15] D. T. Phuc e J. Attikiouzel, "Generalised analysis of Nonmonotonic Lowpass filters with minimum power loss characteristic", Electronics Letters vol. 14, no. 12, pp. 359-360, 1978

[16] Thajchayapong e P. Lomtong, "Transition between a flat magnitude and a sharp cut-off Lowpass filter", International Journal of Electronics vol. 46 no. 1 , pp. $91-95,1979$.

[17] H. Malvar e L. Calôba, "Least-Squares Low-Pass Filters with Nonmonotonic Response", IEEE Transactions on Circuits and Systems vol. 27, no. 12, pp.1270- 1272, 1980.

[18] B. Rakovich e V. Litovski, "Least-squares monotonic Lowpass filters with sharp cutoff', Electronics Letters vol. 9, no. 4, pp. 75-76, 1973.

[19] P. Halpern, “Optimum Monotonic Low Pass Filters" IEEE Transactions on Circuit Theory, vol. CT-16, pp. 240-241, 1969.

[20] J. Kurtz, H. Orchard e G. Temes, "Least-squares Passband filters", IEEE Transactions on Circuit Theory vol. 19, no. 3, pp. 302-304, 1972.

[21] B. Rakovich, "Designing monotonic low-pass filters - comparison of some methods and criteria", International Journal of Circuit Theory and Applications vol. 2, no 3, pp. 215-221, 1974.

[22] B. Djurich e R. Petkovich, "Generalized analysis of optimum monotonic low-pass filters", IEEE Transactions on Circuits and Systems vol. 23, no. 11, pp.647-649, 1976.

[23] B. D. Rakovich e M. V. Popovich, "Explicit expression for the characteristic function of generalized Legendre filters", Circuit Theory and Applications vol. 6, pp. 363-373, 1978. 